\title{
Fear of the COVID-19 and medical liability. Insights from a series of 130 consecutives medico-legal claims evaluated in a single institution during SARS-CoV-2-related pandemic
}

\author{
Matteo $\mathrm{Nioi}^{1, *, \dagger}$, Pietro Emanuele Napoli ${ }^{2, \dagger}$, Gabriele Finco ${ }^{3}$, Roberto Demontis ${ }^{1}$, \\ Maurizio Fossarello², Ernesto d'Aloja ${ }^{1}$
}

${ }^{1}$ Department of Clinical Sciences and Public Health, University of Cagliari, Forensic Medicine Unit, 09124 Cagliari, Italy

${ }^{2}$ Department of Surgical Science, University of Cagliari, Eye Clinic, 09124 Cagliari, Italy

${ }^{3}$ Department of Clinical Sciences and Public Health, University of Cagliari,

Anesthesiology Unit, 09124 Cagliari, Italy

\section{*Correspondence}

nioimatteo@gmail.com

(Matteo Nioi)

$\dagger$ These authors contributed equally.

\begin{abstract}
Objectives: The aim of the current work was to evaluate the frequency and the type of cases of medical liability from a single center in the first ten months of the pandemic as well as to identify critical issues associated with the organization of public health during the coronavirus disease 2019 pandemic.

Methods: We compared 130 cases evaluated for medical liability from March 2020 to January 2021 and compared with 159 cases from March 2019 to January 2020. The cases were divided in four pre-established groups: surgical error, diagnostic/therapeutic errors and nosocomial diseases, delays, and problems related to assistance.

Results: Analysis showed a significant increase in cases due to delay in treatment/hospitalization and shortages in health care of non-autonomous patients $\left[\chi^{2}(1\right.$, $\mathrm{N}=289)=5.6746, p=0.02]$.

Conclusions: The work showed an increase in medical/legal cases regarding nonCOVID-19 emergencies in which the outcome is related to the time of treatment and/or arrival at the hospital. There was also a rise in complaints of deficits in supervision and care for non-autonomous patients. Despite the persistence of preventive measures for the current pandemic, measures should be taken to improve health care in these categories of patients.
\end{abstract}

\section{Keywords}

COVID-19; COVID-19 and medical liability; Medico-legal claims; COVID-19 and forensic pathology; COVID-19 penal shield; COVID-19 risk management; COVID-19 and triage; COVID-19 delay; Surgical error; medical error; Lockdown effects; COVID19 fragile patients; COVID-19 mental health; COVID-19 childbirth; SARS-Cov-2

\section{Introduction}

The analysis of forensic cases concerning medical liability represents a privileged observatory from which to identify early issues in the healthcare organization and to implement rapid corrections to potential biases [1]. On 11 March 2020, WHO declared the pandemic nature of the severe acute respiratory syndrome coronavirus 2 (SARS-CoV-2) outbreak. The related disease was named coronavirus disease 2019 (COVID-19) and spread rapidly around the globe. Until then some areas, due to factors not yet fully understood [2], did not have a very high incidence of the disease. Others like Italy were hit with extreme severity. Doctors have progressively realized that the disease, initially considered as a form of "more severe flu", was in fact a multisystem disease with a severe prognosis [3-5].

Healthcare workers from countries where the pandemic was spreading rapidly suddenly had to fight against a new and unknown virus with a lack of personal protective equipment (PPEs). In this situation, many doctors and healthcare workers (HCWs) developed a human and understandable fear of contracting COVID-19 that affected both the relationship with patients and the practice of the profession.

The rapid expansion of the COVID-19 pandemic resulted in the need to quickly adapt the health policies of different countries. In terms of medical liability strictly linked to the pathology, various health systems have found different solutions; thus, an emerging problem is the effect that the reorganization of facilities has had on the management of "nonCOVID" pathologies. The fear of COVID-19 can manifest itself in different ways: It can concern HCWs, governments, and patients. Several authors addressed the issue of 'indirect' clinical consequences on patients with chronic diseases who have avoided routine scheduled examinations [6-12].

Even from the medical-legal viewpoint, there are still eth- 
ical, scientific, and legal dilemmas which, one year after the pandemic, must necessarily be analyzed $[13,14]$.

The aim of the current work was to analyze the cases of suspected malpractice that came to our attention during the current pandemic in which, although not concerning COVID19 patients, the preventive measures adopted likely had a negative effect.

\section{Methods}

\subsection{Sampling and classification of medico-legal cases}

We evaluated claims for medical liability from March 2019January 2020 and March 2020-January 2021 regardless of whether the assessment was requested by the Public Prosecutor or by Law Firms. The cases for which an evaluation was requested and in which a deviation from guidelines was detected were considered. A simple claim is not necessarily synonymous with the existence of a legally relevant fault. According to a number of Insurance Italian reports, $22.7 \%$ to $50.1 \%$ of cases do not lead to compensation [15-18].

The cases were categorized according to the type of deviation from the ideal conduct identified. The term 'error in surgical technique' (Group A) implies cases with a technical error during the interventions.

The 'error in therapeutic diagnostic management' (Group B) means any deviation from the clinical management of patients prescribed by guideline algorithms. Nosocomial infections were also considered in this group.

The 'delay' group (Group C) means a longer time required than indicated in the guidelines for transfer to hospital, for the evaluation of the onset of symptoms, for the treatment of the underlying pathology compared to what is required by the guidelines, and/or by the good clinical practices. This group included only cases to be treated in emergency or urgency in which the outcome of the disease was time-dependent (acute myocardial infarcts, aortic dissection, strangulationstrangulation of intestinal hernia, cerebral hemorrhage, stroke, acute appendicitis, acute abdomen). Conversely, diagnostic delays that did not require emergency-urgency treatment (diagnosis of neoplastic pathology) were counted among generic treatment errors.

Finally, 'lack of supervision' (Group D) implies cases in which the subjects required continuous support from the caregivers - due to lack of physical or mental autonomy (bedridden patients, elderly patients with cognitive problems, psychiatric patients, patients who are not independent in the activities of daily life) - and a lack of support was the cause of the damage.

\subsection{General analysis}

In our main analysis, we compared the categories of error not influenced by the measures taken during the COVID emergency (Group A and Group B) with those for which the restrictive measures for prevention of COVID-19 may have played a role in the error (Group C and Group D).

\subsection{Sub-analysis of the groups}

Group $\mathrm{C}$ was distinguished from cases in which the delay in accessing the hospital or in the surgical evaluation or in carrying out the intervention was due to the patient's conditions and not COVID-19. For example, the patient required stabilization or those that had a delay with respect to what is foreseen in the guidelines due to organizational-structural problems (unavailability of means of transport, health personnel, surgical rooms within the structure).

In the group examined (cases between March 2020 and January 2021), after a careful check of the clinical documentation in the records, we considered delays attributable to COVID19 those in which there was a concrete application of the guidelines for prevention from SARS-CoV-2 that resulted in a longer times due to COVID-specific changes in protocol. Group D was compared with the control group based on the causes that led to a request for evaluation.

\subsection{Statistical analysis}

Data analysis was performed by Statistical Package for Social Science (SPSS version 21.0). Numerical variables were summarized as mean \pm standard deviation (SD) for parametric data, and nominal data were summarized as percentage. The normality of the data used the Shapiro-Wilk test and Lilliefors test. If data were normally distributed, then parametric statistical tests were used; otherwise nonparametric tests were used.

Cases were subdivided according to the categories. The data were analyzed via a $\chi^{2}$ test. Other specific statistical tests are described as they are encountered in the article. $p$-values less than 0.05 were considered significant.

\section{Results}

\subsection{Sample description}

There were 159 cases from March 2019-January 2020 with 71 females (44.64\%) and 88 males (55.36\%); all were Caucasian. The mean age was 60.24 years [range 1-98; DS 15.6]. As for the categories, 61 cases $(38.4 \%)$ were attributable to a surgical error. The other 63 cases $(39.6 \%)$ were attributable to diagnostic or therapeutic errors/nosocomial infections. Twenty cases $(12.6 \%)$ were attributable to a delay in treatment (diseases for which the outcome was time-dependent such as myocardial infarctions, aortic dissections, hemorrhages, etc.). The other 15 cases $(9.4 \%)$ were attributed to a problem in patient care (especially falls in hospital or injuries caused by patients entrusted to the hospital who had left the hospital without the knowledge of the healthcare workers).

There were 130 cases from March 2020-January 2021 with $57(43.85 \%)$ women and $73(56.15 \%)$ men all of Caucasian race and average age of 59.2 years [range 1-102; DS 16.52]. Surgical errors were reported in 45 cases $(34.6 \%)$. Forty cases $(30.8 \%)$ had diagnostic, therapeutic, or nosocomial origin pathologies (surprisingly no cases of COVID-19). In 25 (19.2\%) cases, the request for evaluation was based on a delay in treatment or access to the hospital. In the remaining 20 cases $(15.4 \%)$, the requests for evaluation were based on a patient care problems (Fig. 1). 


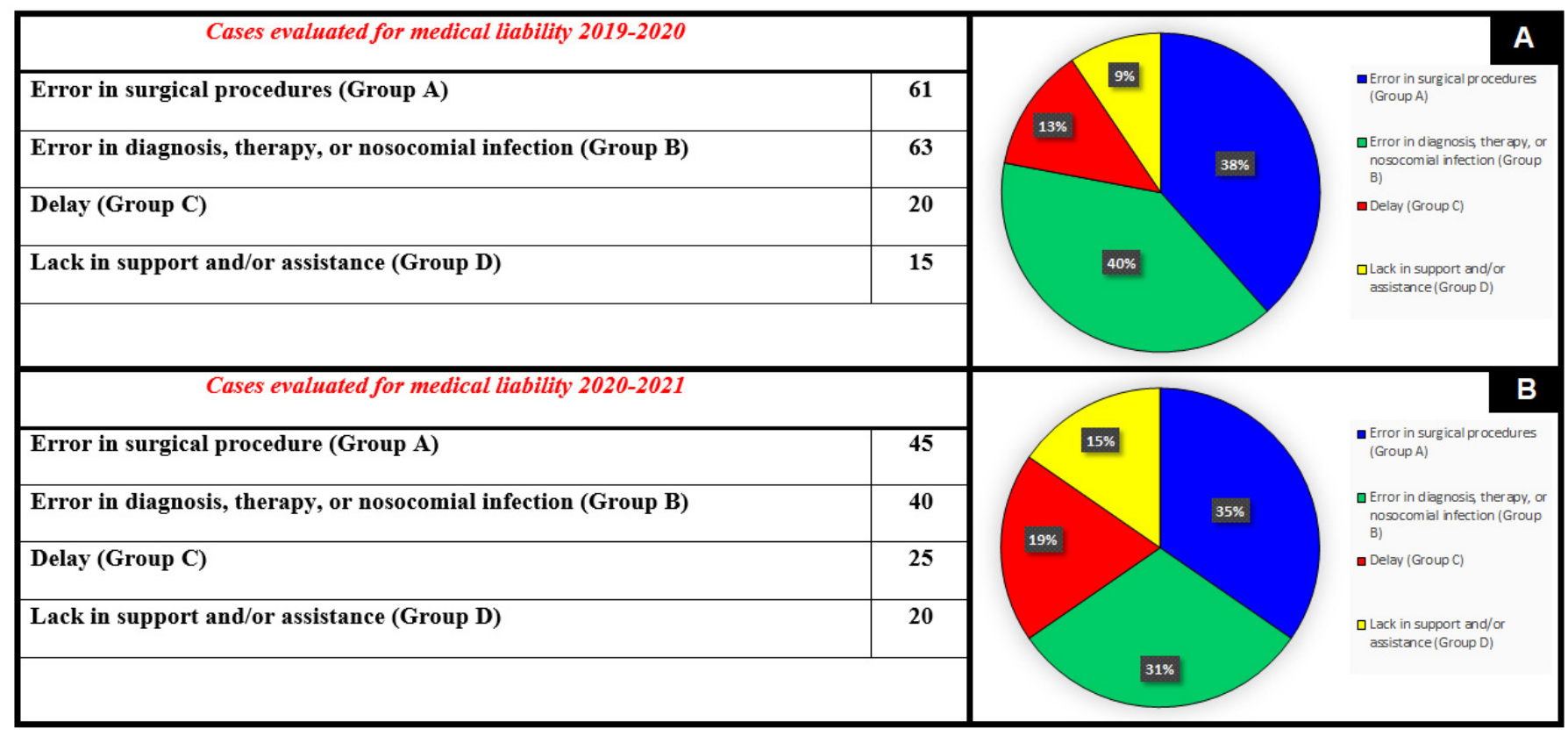

F I G U R E 1. Sample under examination and control sample divided according to the causes that led to the evaluation for any professional liability.

TA B L E 1. Results of general analysis.

\begin{tabular}{|ccc|} 
& Group A + Group B & Group C + Group D \\
\hline INTERVAL 2019-2020 & $124(114.99)[0.71]$ & $35(44.01)[1.85]$ \\
\hline INTERVAL 2020-2021 & $85(94.01)[0.86]$ & $45(35.99)[2.26]$ \\
\hline
\end{tabular}

The observed cell totals, the expected cell totals (in round brackets) and the chi-square statistic for each cell (square brackets) were reported.

\subsection{Results of general analysis}

The cases attributable to groups $\mathrm{A}+\mathrm{B}$ were compared with those attributable to those $\mathrm{C}+\mathrm{D}$. The analysis used a $\chi^{2}$ test with tolerated error fixed at $5 \%(\alpha=0.05)$.

The test results showed a significantly significant increase from March 2020 to January 2021 for groups C and D versus the reference period (March 2019-January 2020). The $\chi^{2}$ test was 5.67. The $p$-value was 0.11 (significant at $p<0.05$ ) (Table 1).

\subsection{Temporal distribution of cases}

The distribution of cases was uniform over time: March-May, June-September, and October-January. There was a decrease in the absolute number of Groups A and B complaints and a simultaneous increase in the absolute number of complaints for cases in groups $\mathrm{C}$ and $\mathrm{D}$ mainly in phase I of the pandemic in Italy (hard lockdown). The absolute number of complaints in the subsequent phases (June-September and October-January) is consistent with what occurred in the control group (Fig. 2).

\subsection{Focus on Groups C and D}

The data showed differences in group C (delay in diagnosing and performing surgical interventions with respect to the time frame indicated in the guidelines) and group D (injuries due to a lack of care). For group $\mathrm{C}$ analysis, we performed a first-level analysis on the 25 cases of the study group and on the 20 cases of the control group distinguishing between cases in which the delay was due to the conditions of the patient and those in which it was due to organizational or structural problems.

Of the 25 cases, in $12(48 \%)$ cases the delay was due to the need for stabilization of the patient following a decompensation that made them not transportable (polytrauma after road accident, need to support vital functions on site). In two (8\%) cases, the event occurred in centers distant from a hospital able to guarantee a surgical/therapeutic intervention $(>1.5 \mathrm{~h})$. In two $(8.0 \%)$ cases, due to the lack of specificity of the symptoms, the patients were referred to specialist cardiological care and died at home due to AMI and intestinal perforation. In nine (36\%) cases, there was a delay with respect to the reference timing, and the course of time-dependent diseases was directly influenced by the implementation of the protocols necessary to prevent the spread of the COVID-19 pandemic. In particular, five of these $(55.56 \%)$ cases led to the activation of the protocol with delayed diagnosis of the underlying pathology: aortic dissection, two cases of AMI, strangulated inguinal hernia, acute appendicitis. These subjects had hyperpyrexia, which required a SARS-Cov- 2 testing after triage according COVID19 guidelines.

In one (11.11\%) case (bacterial pneumonia), the presence of dyspnea meant that the COVID-19 protocol was activated, but a medical vehicle arrived on site three hours after the first call and the patient died during transport to the hospital. (The medical vehicles were engaged in other aid and the volunteers 


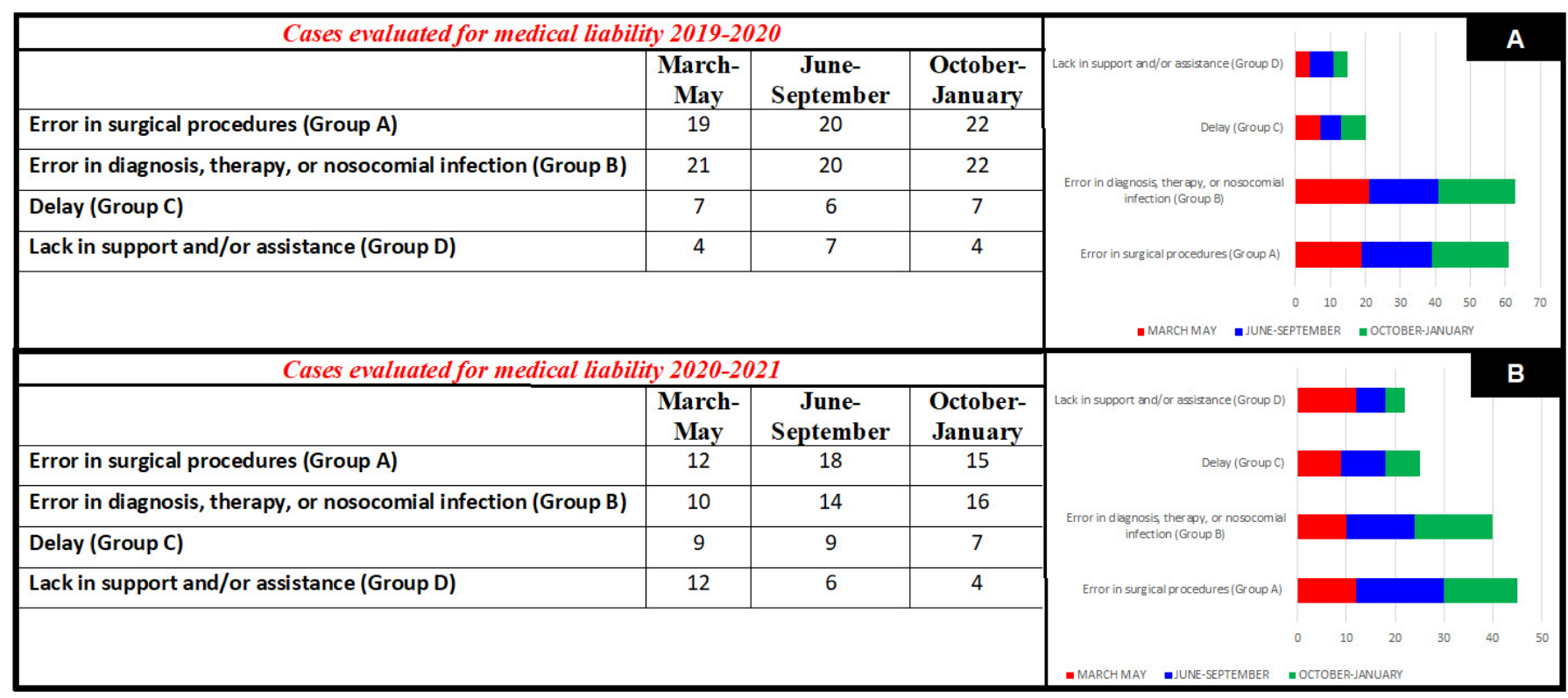

F I G U R E 2. Chronological distribution in the different phases of the year. Cases in 2019-2020 (A) divided according to time intervals corresponding in 2020-2021 (B) to the tight lockdown period (March-May), decrease in restrictions (June-September), and return to moderate restrictions (October-January).

did not have the PPE equipment necessary to transport the patient). In another (11.11\%) case, the lack of operating rooms due to the need to sanitize the environment led to a two-day delay of an operation for abdominal aortic aneurysms of an already hospitalized patient. During this period, there was a sudden precipitation of symptoms leading to death. In two cases $(22.22 \%)$, the nearest hospital was a COVID-19 center, and the patients were referred to another center after a pretriage from which non-specific signs emerged (epigastric pain and non-specific abdominal pain which later turned out to be a fissured aortic aneurysm MI).

Out of the nine cases examined, the delay in the treatment of pathologies in which the outcome is time-dependent led to death. In one (appendicitis) the patient came to the attention of a surgeon more than 48 hours after the onset of symptoms and a laparoscopic intervention was required. Of the 20 patients in the control group, 15 cases $(75 \%)$ had delay due to poor stability of the patient for polytrauma ( 8 cases) or for different medical conditions. These situations made the patients nontransportable prior to stabilization.

In three $(15 \%)$ cases, the patient suffering from atypical symptoms (in one case characterized by abdominal pain and headache in others) was sent home pending specialist examinations. In two $(10 \%)$ cases, the delay was due to the coordination between rescues or to the distance of the hospital structure capable of managing a specific type of pathology (Fig. 3).

With regards to assessments requested for alleged "care shortages", the absolute number of requests regarding falls in hospital (nine cases) remained unchanged even if the patient denounced them for this cause there was a decrease in terms of percentage ( $64 \%$ vs. $45 \%)$. There was a slight decrease for injuries in non-autonomous patients who went around the wards [five $(29 \%)$ vs. two (10)]. On the other hand, there was a slight increase in complaints for patients who died in the facilities without direct assistance from health personnel [one $(7 \%)$ vs. two $(10 \%)]$. Unlike in 2019 , there was one $(5 \%)$ complaint for a patient who committed suicide in the health facility and six (30\%) requests for evaluation for patients in whom pressure ulcers developed (Fig. 4).

\section{Discussion}

The data show a decrease in the raw number of cases that came to our assessment for alleged medical irresponsibility from March 2020 to January 2021 versus the control group (130 vs. 159). This finding is logically consistent with the decrease in the absolute number of non-COVID-19 related hospitalizations in Italy over this period [19-22].

Other literature shows that patients were afraid of contracting the virus leading to a lower request for healthcare services/hospitalizations - particularly in the period of hard lockdown (March-May 2020). This factor certainly contributed to a lower afflux of such categories of patients to hospital and to contentious relationship between patients and HCWs. On the other hand, the hard lockdown led to a worsening of the conditions of non-autonomous patients. Counterintuitively, there was no contentious relationship between HCWs and COVID-19 nor the wave of reports against HCWs [23, 24].

Moreover, there was a statistically significant increase in requests concerning particular categories of events against a decrease in the overall number of requests for evaluation for medical responsibility: complaints/requests for evaluation due to delay in the management of acute life-treating disease diseases other than COVID-19, and the causes/requests for evaluation for a lack of assistance by figures legally called to take care of non-autonomous patients during hospitalization.

The pandemic has led to important critical issues requiring action especially fear of disease and the preventive measures 


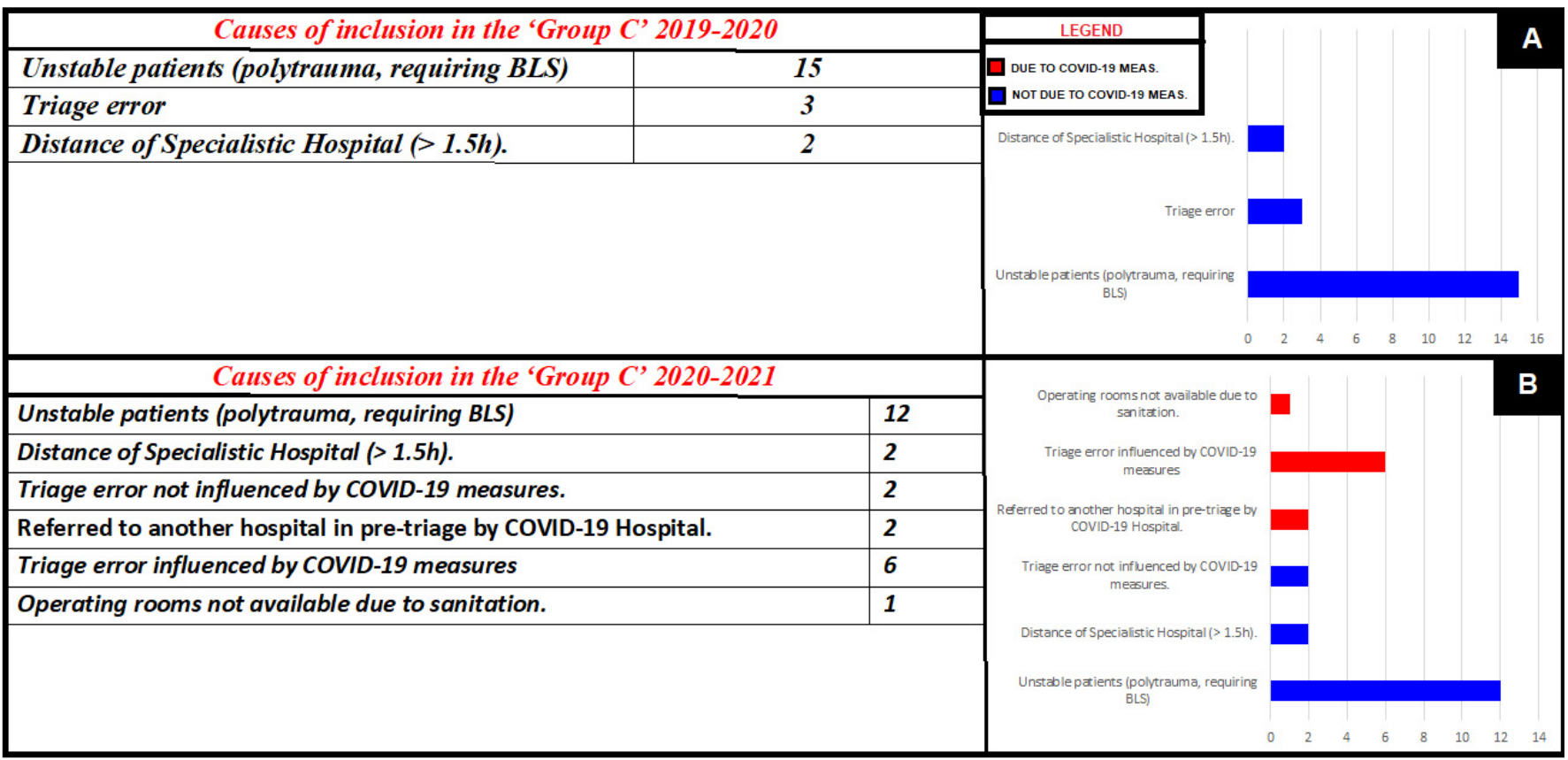

F I G U R E 3. Group C (delay) sub-analysis. (A) Data from March 2019-January 2020. (B) Data from March 2020-January 2021. In red triage errors influenced by COVID-19 related measures. In blue triage errors not influenced by COVID-19 related measures.

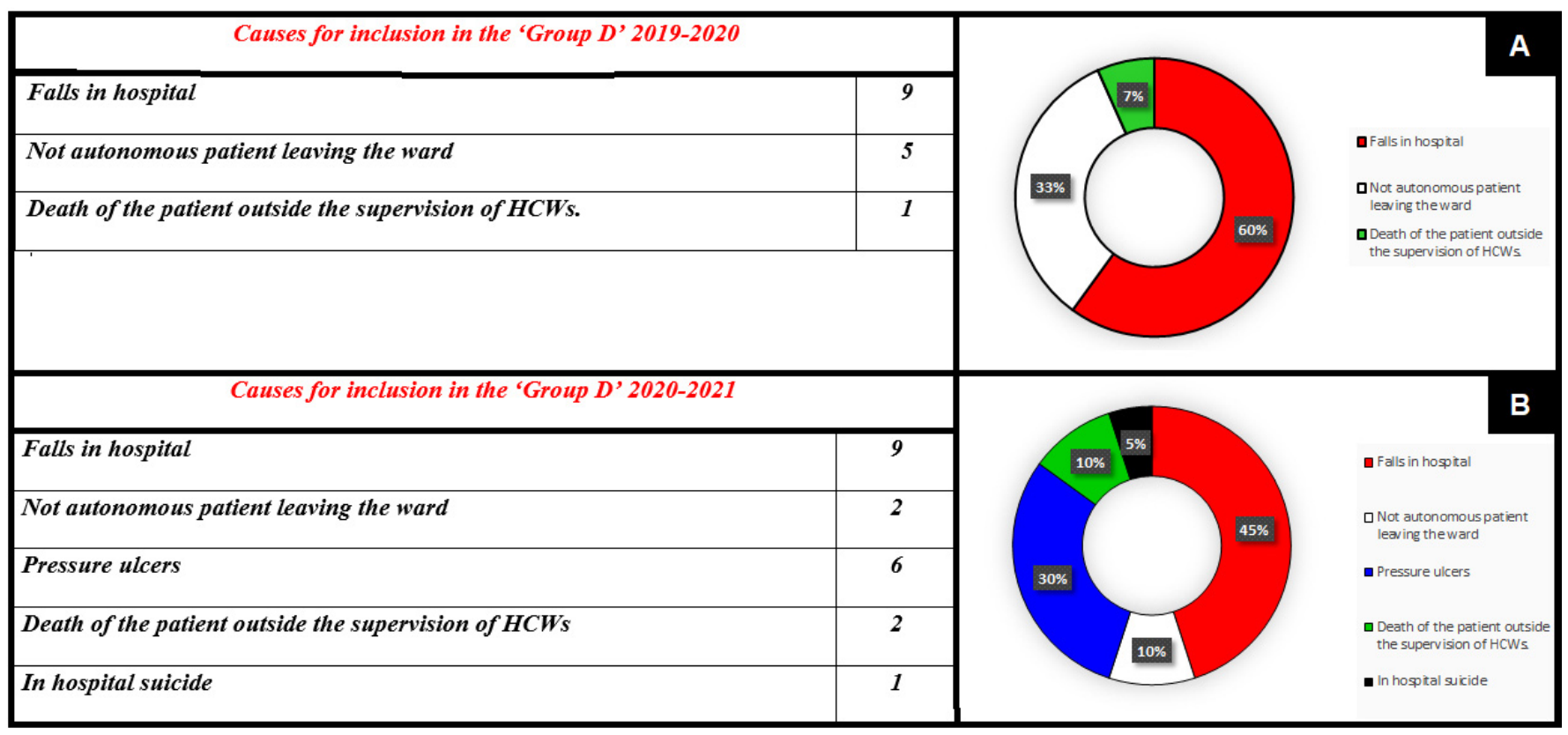

F I G U R E 4. Group D (assistance) sub-analysis. (A) Data from March 2019 to January 2020. (B) Data for the interval March 2020 to January 2021.

required. The biggest problem was error in triage. The presence of atypical symptoms that could be linked to COVID-19 such as fever and dyspnea meant that the appropriate COVID19 protocol was activated thus delaying the execution of necessary diagnostic tests or the early execution of an adequate therapeutic procedure [25]. The effects of a "COVID-19 centric triage" may somehow be deleterious and life-treating although still necessary. A return to prioritization based on the severity of the pathology and on an a priori sound risksbenefits analysis seems to be advisable.
In some cases, the dedication of peripheral hospitals to the treatment of patients with COVID-19 diverted the afflux of emergencies to other more distant hospitals. This event weighed on the treatment of severe conditions in which time was an important outcome factor. One case emphasized difficulties related to the sanitation of the surgical room, which caused an operation to be postponed. This patient was already under observation and apparently had stabile conditions that unexpectedly precipitated and led to his death [26].

The second problem is from "fragile patients" (i.e., not 
self-sufficient and/or psychiatric). In these categories, even the omission of "assistance activities" - not mandatory for autonomous patients - can lead to rapidly life-treating worsening [27]. Such cases were frequent in the very first phase of the pandemic (March-May) when the action of auxiliary HCWs was not supported by the caregivers of patients who were prevented from accessing health facilities. Caregivers were unable to support people who are not self-sufficient. Furthermore, individuals with unique needs (e.g., women in childbirth, the institutionalized elderly, or psychiatric patients) need support [31-37]. Interestingly, one patient with depression committed suicide [38-40].

Overall, the pandemic has given rise to new cases of medical liability. As far as delays are concerned, a complete evaluation of the patient is important and can highlight cases that need to be treated in an emergency. More than a year after the onset of the SARS-Cov-2 epidemic, it is no longer acceptable for triages to be carried out according to a "COVID-19-centric" principle. An improvement in the situation could be produced by entrusting the evaluation of triage. Currently, this is delegated to the nursing staff in Italy and to doctors able to grasp the symptomatic subtleties [41, 42].

Clearly, after the initial uncertainty, the system will have offer rapid and effective responses to patients who come to the hospital for emergencies other than COVID-19 through adequate logistical-organizational support. Also, sanitation of the surgical rooms should be fast to perform emergency interventions. Vaccinations and the availability of PPE can help fragile patients access caregivers or qualified personnel for safe and adequate physical and emotional support.

\section{Conclusions}

In conclusion, eliminating the COVID-19 fear that emerged from the examined cases could have a triple benefit: nonautonomous and emergency patients would have optimal treatment; doctors would have peace of mind; and the health system would drastically reduce potential litigation saving valuable resources useful for managing the pandemic.

\section{AUTHOR CONTRIBUTIONS}

MN, EdA, GF and PEN conceived of the presented idea. EdA, PEN, MN and RD developed the theory. MN wrote the manuscript in consultation with EdA, PEN, GF and MF.

\section{ETHICS APPROVAL AND CONSENT TO PARTICIPATE}

Formal ethical approval by the Independent Review Board was not required in accordance with the policy of our Institution for non-pharmacological studies. Being the focus of the study on the retrospective evaluation of the frequency and the type of cases of medical liability, only a preliminary ethical evaluation on soundness of the scientific hypothesis and on the respect of the principles of the Declaration of Helsinki (and further modifications) was carried out. For this reason, an ethical code number was not assigned to the study but only a 'nihil obstat' was sent to the principal investigator on 16 February 2021.

\section{ACKNOWLEDGMENT}

Thanks to all the peer reviewers for their opinions and suggestions and to the physicians in training for their precious collaboration.

\section{FUNDING}

This research received no specific grant from any funding agency in the public, commercial, or not-for-profit sectors. At the time of data acquisition, the authors were employed at University of Cagliari, Italy, where the study was conducted.

\section{CONFLICT OF INTEREST}

The authors declare no conflict of interest.

\section{DATA AVAILABILITY}

The data used to support the findings of this study are available from the corresponding author upon request at the conclusion of the judicial procedure relating to each individual case.

\section{REFERENCES}

[1] Hickson GB. Patient Complaints and Malpractice Risk. Journal of the American Medical Association. 2002; 287: 2951-2957.

[2] Napoli PE, Nioi M. Global spread of coronavirus disease 2019 and malaria: an epidemiological paradox in the early stage of a pandemic. Journal of Clinical Medicine. 2020; 9: 1138.

[3] Napoli PE, Nioi M, d'Aloja E, Fossarello M. The ocular surface and the coronavirus disease 2019: does a dual 'ocular route' exist? Journal of Clinical Medicine. 2020; 9: 1269.

[4] Akhmerov A, Marbán E. COVID-19 and the heart. Circulation Research. 2020; 126: 1443-1455.

[5] Puelles VG, Lütgehetmann M, Lindenmeyer MT, Sperhake JP, Wong $\mathrm{MN}$, Allweiss L, et al. Multiorgan and renal tropism of SARS-CoV-2. New England Journal of Medicine. 2020; 383: 590-592.

[6] Wright A, Salazar A, Mirica M, Volk LA, Schiff GD. The invisible epidemic: neglected chronic disease management during COVID-19. Journal of General Internal Medicine. 2020; 35: 2816-2817.

[7] Napoli PE, Nioi M, Fossarello M. The "quarantine dry eye": the lockdown for coronavirus disease 2019 and its implications for ocular surface health. Risk Management and Healthcare Policy. 2021; 14: 16291636.

[8] Nouri S, Khoong EC, Lyles CR, Karliner L. Addressing equity in telemedicine for chronic disease management during the Covid-19 pandemic. NEJM Catalyst Innovations in Care Delivery 2020; 1.3.

[9] Louvardi M, Pelekasis P, Chrousos GP, Darviri C. Mental health in chronic disease patients during the COVID-19 quarantine in Greece. Palliative and Supportive Care. 2020; 18: 394-399.

[10] DeJong C, Katz MH, Covinsky K. Deferral of care for serious nonCOVID-19 conditions. JAMA Internal Medicine. 2021; 181: 274.

[11] Chudasama YV, Gillies CL, Zaccardi F, Coles B, Davies MJ, Seidu S, et al. Impact of COVID-19 on routine care for chronic diseases: a global survey of views from healthcare professionals. Diabetes \& Metabolic Syndrome: Clinical Research \& Reviews. 2020; 14: 965-967.

[12] Mauro V, Lorenzo M, Paolo C, Sergio H. Treat all COVID 19-positive patients, but do not forget those negative with chronic diseases. Internal and Emergency Medicine. 2020; 15: 787-790.

[13] Nioi M, Napoli PE, Fossarello M, d'Aloja E. Autopsies and asymptomatic 
patients during the COVID-19 pandemic: balancing risk and reward. Frontiers in Public Health. 2020; 8: 595405.

[14] Bogdanović M, Atanasijević T, Popović V, Mihailović Z, Radnić B, Durmic $\mathrm{T}$. Is the role of forensic medicine in the covid-19 pandemic underestimated? Forensic Science, Medicine and Pathology. 2021; 17: 136--138.

[15] Gualniera P, Mondello C, Scurria S, Oliva A, Grassi S, Pizzicannella J, et al. Experience of an italian hospital claims management committee: a tool for extrajudicial litigations resolution. Legal Medicine. 2020; 42: 101657.

[16] MARSH. Report MEDMAL -SINTESI Studio sull'andamento del rischio da medical malpractice nella sanità italiana pubblica e privata. 9th Ed. 2018 Available at: https://www. marsh.com/content/dam/marsh/ Documents/PDF/it/it/Medmal_2018.pdf (Accessed: 1 May 2021).

[17] MARSH. Report MEDMAL -SINTESI Studio sull'andamento del rischio da medical malpractice nella sanità italiana pubblica e privata. 10th Ed. 2019 Available at: https://www. marsh.com/content/dam/marsh/ Documents/PDF/it/it/Medmal_2019.pdf (Accessed: 1 May 2021).

[18] MARSH. Report MEDMAL -SINTESI Studio sull'andamento del rischio da medical malpractice nella sanità italiana pubblica e privata- EXECUTIVE SUMMARY 11th Ed. 2020 Available at: https://www.marsh.com/content/dam/marsh/Documents/PDF/ it/it/Marsh_Medmal_report_2020_web.pdf (Accessed: 1 May 2021).

[19] Blecker S, Jones SA, Petrilli CM, Admon AJ, Weerahandi H, Francois F, et al. Hospitalizations for chronic disease and acute conditions in the time of COVID-19. JAMA Internal Medicine. 2021; 181: 269-271.

[20] Secco GG, Zocchi C, Parisi R, Roveta A, Mirabella F, Vercellino M, et $a l$. Decrease and delay in hospitalization for acute coronary syndromes during the 2020 SARS-CoV-2 pandemic. Canadian Journal of Cardiology. 2020; 36: 1152-1155

[21] Findling MG, Blendon RJ, Benson JM. Delayed care with harmful health consequences - reported experiences from national surveys during coronavirus disease 2019. JAMA Health Forum. 2020; 1: e201463.

[22] De Filippo O, D'Ascenzo F, Angelini F, Bocchino PP, Conrotto F, Saglietto A, et al. Reduced rate of hospital admissions for ACS during Covid-19 outbreak in Northern Italy. New England Journal of Medicine. 2020; 383: 88-89.

[23] d'Aloja E, Finco G, Demontis R, Napoli PE, Fossarello M, Nioi M. COVID-19 and medical liability: Italy denies the shield to its heroes. EClinicalMedicine. 2020; 25: 100470

[24] Nioi M, Napoli PE, Lobina J, Fossarello M, d'Aloja E. COVID-19 and Italian healthcare workers from the initial sacrifice to the mRNA vaccine: pandemic chrono-history, epidemiological data, ethical dilemmas, and future challenges. Frontiers in Public Health. 2020; 8: 591900.

[25] Solomon MZ, Wynia MK, Gostin LO. Covid-19 crisis triage—optimizing health outcomes and disability rights. New England Journal of Medicine. 2020; 383: e27.

[26] Napoli PE, Nioi M, d'Aloja E, Fossarello M. Safety recommendations and medical liability in ocular surgery during the COVID-19 pandemic: an unsolved dilemma. Journal of Clinical Medicine. 2020; 9: 1403.

[27] Nioi M, Napoli PE, Ferreli F. Fatal iatrogenic pituitary apoplexy after surgery for neuroophthalmological disorder. Anesthesiology. 2019; 130: 822.

[28] Elshafeey F, Magdi R, Hindi N, Elshebiny M, Farrag N, Mahdy S, et al. A systematic scoping review of COVID-19 during pregnancy and childbirth. International Journal of Gynecology \& Obstetrics. 2020; 150: 47-52.

[29] Ahlers-Schmidt CR, Hervey AM, Neil T, Kuhlmann S, Kuhlmann Z. Concerns of women regarding pregnancy and childbirth during the COVID-19 pandemic. Patient Education and Counseling. 2020; 103: 2578-2582.

[30] Souto SPA, Albuquerque RS, Prata AP. Fear of childbirth in time of the new coronavirus pandemic. Revista Brasileira de Enfermagem. 2020; 73 : e20200551.

[31] Arbaje A. Coronavirus and COVID-19: caregiving for the Elderly. 2020 Avaialble at: https://www.hopkinsmedicine.org/health/ conditions-and-diseases/coronavirus/coronaviruscaregiving-for-the-elderly (Accessed: 1 May 2021).

[32] Araújo MPD, Nunes VMA, Costa LA, Souza TA, Torres GV, Nobre TTX Health conditions of potential risk for severe Covid-19 in institutionalized elderly people. PLoS ONE 2021; 16:e0245432.

[33] Banerjee D. 'Age and ageism in COVID-19': elderly mental health-care vulnerabilities and needs. Asian Journal of Psychiatry. 2020; 51: 102154.

[34] Araújo PO, Freitas MYGS, Carvalho ESS, Peixoto TM, Servo MLS, Santana LDS, et al. Institutionalized elderly: vulnerabilities and strategies to cope with Covid-19 in Brazil. Investigación y Educación en Enfermería. 2021; 39: e07.

[35] Bojdani E, Rajagopalan A, Chen A, Gearin P, Olcott W, Shankar V, et al COVID-19 pandemic: impact on psychiatric care in the United States. Psychiatry Research. 2020; 289: 113069 .

[36] Steardo L, Steardo L, Verkhratsky A. Psychiatric face of COVID-19. Translational Psychiatry. 2020; 10: 261.

[37] Li LZ, Wang S. Prevalence and predictors of general psychiatric disorders and loneliness during COVID-19 in the United Kingdom. Psychiatry Research. 2020; 291: 113267.

[38] Gunnell D, Appleby L, Arensman E, Hawton K, John A, Kapur N, et al. Suicide risk and prevention during the COVID-19 pandemic. Lancet Psychiatry. 2020; 7: 468-471.

[39] Sher L. The impact of the COVID-19 pandemic on suicide rates. QJM: An International Journal of Medicine. 2020; 113: 707-712.

[40] Klomek AB. Suicide prevention during the COVID-19 outbreak. The Lancet Psychiatry. 2020; 7: 390.

[41] Bambi S, Ruggeri M, Sansolino S, Gabellieri M, Tellini S, Giusti M, et al. Emergency department triage performance timing. a regional multicenter descriptive study in Italy. International Emergency Nursing. 2017; 29: 32-37.

[42] Weston V, Jain SK, Gottlieb M, Aldeen A, Gravenor S, Schmidt MJ, et al. Effectiveness of resident physicians as triage liaison providers in an academic emergency department. Western Journal of Emergency Medicine. 2017; 18: 577

How to cite this article: Matteo Nioi, Pietro Emanuele Napoli, Gabriele Finco, Roberto Demontis, Maurizio Fossarello, Ernesto d'Aloja. Fear of the COVID-19 and medical liability. Insights from a series of 130 consecutives medico-legal claims evaluated in a single institution during SARS-CoV-2-related pandemic. Signa Vitae. 2021;17(4):79-85. doi:10.22514/sv.2021.098. 